\title{
Evaluación y participación social en el marco de la gerencia pública necesaria
}

\author{
Cardozo Brum, Myriam*
}

\section{Resumen}

América Latina tiene importantes insuficiencias en sus sistemas de evaluación gubernamental y de participación, México no escapa de esta situación. El presente artículo tiene por objetivo analizar el proceso de evaluación de la acción pública y la participación social mexicanas, en el contexto de la gerencia pública. Se analizan 31 programas sociales evaluados, para ello se emplea el método de análisis documental complementado con entrevistas a diferentes actores involucrados. Las variables estudiadas fueron: organización del sistema de evaluación, recursos utilizados, fuentes de información y tratamiento de datos, contenido de la evaluación y participación de los beneficiarios y de organizaciones sociales. Se concluye que México está muy lejos de contar con el nivel de evaluación y participación que pregona el enfoque de la gerencia pública, se han hecho importantes avances en la evaluación de programas gubernamentales, pero aún presenta problemas por resolver y no ha desarrollado mecanismos efectivos de participación social.

Palabras clave: Evaluación, participación, gerencia pública, México.

\section{Social evaluation and participation within the framework of necessary public management}

\begin{abstract}
Latin America has significant deficiencies in its systems of governmental management and participation. Mexico is not an exception. The objective of this article is to analyze the process of the evaluation of Mexican public action and social participation within the context of public management. Thirty-one evaluated social programs are analyzed, using documentary analysis in combination with interviews with the involved actors. The variables studied were: organization of the evaluation system, resources utilized, sources of information and data treatment, content of the evaluation, and participation on the part of the beneficiaries and the social organizations involved. The conclusion is that
\end{abstract}

\section{Recibido: 04-09-02. Aceptado: 05-01-17}

Docente e investigadora del Departamento de Política y Cultura, Universidad Autónoma Metropolitana (Xochimilco). E-mail: mcardozo@correo.xoc.uam.mx 
Mexico is far from achieving the levels of participation and evaluation which are proposed. Important advances have been made in the evaluation of public programs, but there are still many problems and effective mechanisms of social participation have not been developed.

Key words: Evaluation, participation, public management, Mexico.

\section{Introducción}

América Latina presenta importantes insuficiencias en sus sistemas de evaluación gubernamental y de participación social promovidos desde la gerencia pública, parte de este trabajo se dedica a discutir esta situación.

En el caso concreto de México, los programas gubernamentales han comenzado su evaluación externa a partir del año 2001, por decisión de la Cámara de Diputados. Por otro lado, el Plan Nacional de Desarrollo (PND) y el Programa Nacional de Desarrollo Social (PNDS) para el período 2001-2006, así como el discurso gubernamental, enfatizan la importancia de la participación ciudadana.

Sin embargo, en la práctica, la evaluación aún enfrenta diversas dificultades, entre las que resaltan las relativas a: organización, recursos, información, tratamiento técnico de datos, medición de impactos, difusión de resultados y la gran ausencia de participación social.

En el presente trabajo se analiza el discurso y el desarrollo práctico de la evaluación y la participación social en el marco de la gerencia pública latinoamericana, poniendo énfasis en el estudio de la situación mexicana a través del análisis de 31 evaluaciones a programas sociales realizadas en el 2002.

\section{Evaluación y participación en el contexto de la gerencia pública}

La gerencia pública (traducción habitual de public management), interesada en la función directiva del proceso de gestión pública, pone énfasis en la búsqueda de la eficiencia y los asuntos ligados a liderazgo y capacidad de mando.

Mientras los términos de gestión y gerencia pública llevan mucho tiempo utilizándose en países como Venezuela, en México aparecen enlazados con el advenimiento del New Public Management (NPM), traducido en nuestro medio como Nueva Gerencia o Nueva Gestión Pública. Este enfoque fue desarrollado a partir de las experiencias de reforma del Estado instrumentadas en varios países sajones, particularmente Nueva Zelanda, Reino Unido y Australia, y retomadas parcialmente en múltiples países de América Latina. Se trata de un movimiento aún en curso, que ha recibido un decidido apoyo de la Organización para la Cooperación y el Desarrollo Económico (OCDE), El Fondo Monetario Internacional (FMI) y el Banco Mundial (BM).

EI NPM se presenta como políticamente neutro, impulsa un modelo de pretensión universal caracterizado por políticas de cambio radical, retoma las críticas realizadas al modelo burocrático (poca 
efectividad de sus resultados) provenientes del Public Choice (Escuela de la Elección Pública) y del Neoinstitucionalismo Económico, y promueve una mayor racionalidad en la toma de decisiones con la finalidad de alcanzar eficacia, economía y eficiencia (las llamadas tres E's) en la gestión pública.

Impulsa orientaciones hacia aspectos como: reducción del tamaño de la burocracia, descentralización, jerarquías aplanadas, cooperación público - privado, apertura a la competencia, privatización, utilización de técnicas de la gerencia privada, flexibilización, simplificación de trámites, desregulación, democratización, enfoque proactivo de problemas, relación costos, calidad, satisfacción y empoderamiento del cliente, orientación a resultados, evaluación del desempeño, utilización de incentivos, rendición de cuentas, entre otros.

Sin embargo, resulta importante subrayar la idea de que gestión y gerencia pública son campos preexistentes al nacimiento del NPM y que, a su interior, existen enfoques distintos y hasta opuestos a éste en algunos aspectos, como el defendido por Kliksberg (1989 y 1994) que no admite la crítica generalizada a la ineficiencia del sector público y lejos de tender a equiparar la administración pública con la privada, enfatiza sus diferencias, incluso las de la gerencia social frente a la gerencia económica, con su particular papel de lo político, la preocupación por la articulación (no subordinación) de las políticas económicas y sociales, los procesos de negociación, la priorización de los intereses de las grandes mayorías empobrecidas, la aplicación del enfoque de políticas públicas, el mayor énfasis en la participación como mecanismos de control social y el desarrollo de programas sociales masivos.

Puesto que la acción pública que impulsa el NPM fundamenta su contenido en los principios de responsabilidad y capacidad de respuesta, y su forma, en la transparencia y la participación social (Bañón, 2003), en este sentido el NPM estimula fuertemente la evaluación gerencial a todos los niveles como una necesidad de buena gestión.

En nuestro trabajo comenzaremos por analizar la importancia de la evaluación de la acción pública y la participación social, contrastando el discurso reinante en nuestros países con la realidad de su aplicación. Para ello se retomarán los antecedentes recientemente publicados sobre algunos países latinoamericanos en materia de evaluación, a los que se agrega el estudio del caso mexicano, que constituye el centro de la reflexión.

Si bien se conocen antecedentes de evaluación en la antigüedad (China en el siglo XX A.C. o Grecia en el siglo V A.C.), en la época moderna estos reaparecen en el siglo XIX, en Inglaterra, en la evaluación de servicios públicos, y, a inicios del siglo XX, en EUA, en la evaluación del aprendizaje educativo.

Los estudios evaluativos en el último país citado reconocen como antecedente el trabajo realizado por Alfred Binet en 1904, quien introdujo el experimentalismo, sobre la base de la psicología, para desarrollar su trabajo de evaluación en el sector de Instrucción Pública. En 1912, Starch y Elliot desarrollaron las primeras evaluaciones de los servicios públicos, contando con un grupo que había recibido un entrenamiento especial y otro que 
funcionó como control. Este enfoque respondía a los supuestos intelectuales de inicios del siglo XX (paradigma positivista), en que la mayoría de los investigadores confiaban en el carácter objetivo de sus observaciones, en la neutralidad axiológica de los instrumentos utilizados, y la utilización de la medición como condición de cientificidad (Monnier, 1995).

Actualmente, se considera que la evaluación es útil al gobierno para alcanzar un mejor diseño de políticas y también útil a los ciudadanos para controlar al gobierno. La evaluación se vuelve herramienta de formación individual y de participación social, de aprendizaje social, de rendición de cuentas y control parlamentario, de mejoramiento de la gestión pública, de la política del gobierno, y de validación del sistema social e ideológico.

La mencionada participación ciudadana permitiría crear condiciones sociales para el ejercicio de la ciudadanía, mediante la promoción de la organización de individuos en torno a intereses y la creación de condiciones para que la comunidad pueda defenderlos, reivindicarlos, articularlos, hacer alianzas y determinar las políticas públicas (Licha, 1998). Es considerada un elemento fundamental del enfoque de gerencia social, promovida por el Banco Interamericano de Desarrollo (BID), destinada a contribuir al fortalecimiento de la democracia, la igualdad social y al desarrollo de proyectos sociales con resultados eficaces para la superación de problemas, al mismo tiempo que enfrenta fuertes resistencias e intereses (Kliksberg, 2000).

La combinación de evaluación y participación da lugar, en el contexto francés, al enfoque pluralista, sostenido por
Monnier (1995). Dicha actitud se fundamenta en el reconocimiento de la diversidad de sistemas de valores que coexisten en el seno de la sociedad y se construye a partir de los conflictos sociales emergentes, lo que lleva al evaluador a actuar como conciliador entre las partes en desacuerdo, en búsqueda de una solución satisfactoria, no óptima, similar a la aplicación de "círculos de calidad" en la industria. Monnier (1995) subraya, además, que la utilidad social de una evaluación depende, no sólo de la metodología empleada, sino de dimensiones políticas como el acceso a la información, la existencia de condiciones que favorezcan la utilización de sus conclusiones y la credibilidad de la misma para los distintos actores, determinada por la legitimidad del proceso seguido, ya que ninguna evaluación puede considerarse neutral, como lo ha sostenido el enfoque tradicional, de corte positivista.

En América Latina múltiples trabajos señalan el limitado avance de la evaluación en los países de la región:

Sulbrandt (1993) consideraba que la mayoría de las políticas y los programas públicos de América Latina (AL) no se evaluaban, especialmente los programas sociales, y que, salvo excepciones, la evaluación no había conducido a provocar ningún cambio en el manejo de los programas ni en el aprendizaje de los ciudadanos.

Brown (2001) señala que en los países en desarrollo, AL y el Caribe en particular, los organismos ejecutores raras veces evalúan los resultados de su gestión, siendo ésta promovida casi siempre por los entes donantes (BM, BID, entre otros) para satisfacer sus propios 
requisitos de responsabilidad fiscal. Plantea que la falta de evaluación de políticas y programas impide una mayor utilidad de los presupuestos por programas y por resultados y provoca importantes costos por falta de aprendizaje y repetición de errores.

De igual manera, Ospina (2001) considera que, salvo en los casos de Chile, Colombia y Costa Rica, AL no ha avanzado en procesos de evaluación sistemática de la administración pública y en la utilización de sus resultados en sus cuatro niveles: políticas públicas, programas públicos, organizaciones públicas y desempeño de los empleados públicos.

En un reciente trabajo, Cunill y Ospina (2003) analizan los casos de los países identificados por Ospina en el trabajo anterior y agregan el de Uruguay, en un intento por medir los actuales esfuerzos de evaluación de la gestión pública en los procesos de modernización y democratización del Estado y la administración pública en la región, en los cuatro casos en que existen iniciativas sólidas y herramientas de evaluación en operación ${ }^{1}$. Identifican las diferencias y semejanzas de los distintos sistemas diseñados y su impacto en el mejoramiento de dicha gestión, con la finalidad de difundir estas experiencias que pueden servir como oportunidad de aprendizaje para otros países. En el nivel meso, de evaluación de organizaciones y programas gubernamentales, destacan los avances del caso chileno.
De su trabajo extraemos los principales antecedentes que se relacionan con la participación en la evaluación de la gestión pública en tres de los países citados:

- En 1994, Colombia realizó su primera propuesta de Sistema Nacional de Evaluación de Resultados de la Gestión Pública (SINERGIA), como herramienta para generar un cambio en la cultura de administración pública, abriendo la gestión a los ciudadanos, al ofrecerles amplia información (accountability). Este sistema careció del apoyo político necesario como consecuencia de la crisis de legitimidad que vivía el gobierno, pero fue validado por el BM y promovido como modelo ejemplar,

- en 1998, Uruguay aprobó su Ley de Rendición de Cuentas, y

- en el año 2000, Costa Rica incorporó a su nueva Constitución la evaluación por resultados y la rendición de cuentas como principios inspiradores de la institucionalidad democrática.

Como puede verse, los tres casos nacionales previos muestran avances exclusivamente en materia de rendición de cuentas, mientras Chile se ha limitado a manifestar su intención de incorporar a la ciudadanía al diseño y evaluación de las políticas y programas públicos. Costa Rica comienza a considerar que la legitimidad en el diseño de indicadores depende de la participación ciudadana.

Como incentivo a las buenas prácticas, los cuatro países utilizan la difusión pública de los resultados de las evaluatrabajo citado. 
ciones de desempeño institucional. Las vías de divulgación generalizada son las páginas web institucionales, Costa Rica utiliza también la conferencia de prensa sobre el tema. En Uruguay, la principal forma de acceso a esta información es la prensa, pero ésta le ha prestado muy poca atención al tema. Esta limitación se agudiza debido al lenguaje excesivamente técnico en que se redactan los documentos de difusión.

En Colombia, Uruguay y Chile, aunque la información se publique en INTERNET, no hay evidencia de que los ciudadanos accedan a ella, lo que Costa Rica ha resuelto, en parte, con sus publicaciones en la prensa.

En cambio, en los últimos cinco años se han fortalecido las organizaciones de la sociedad civil especializadas en la evaluación de la gestión pública, lo que lamentablemente no ha sucedido en el caso de los legisladores.

\section{La experiencia mexicana}

Muchos programas sociales han sido desarrollados en México, especialmente durante el siglo pasado, a cargo de diversas secretarías de estado, organismos descentralizados y empresas de participación estatal mayoritaria. Desde 1917, hubo intentos por crear un organismo que midiera, siquiera parcialmente, los resultados de la gestión gubernamental, poniendo énfasis en el manejo de los recursos. Sin embargo, cada seis años un nuevo gobierno reorientaba los programas gubernamentales para ponerles su sello propio, sin preocuparse por evaluar lo hecho por el anterior.

De esta forma, lo realizado en materia de evaluación fue poco, parcial y es- tuvo a cargo de diversas instancias como las Secretarías del Patrimonio Nacional, de la Presidencia y de Hacienda y Crédito Público (SHCP), desconociéndose realmente los niveles de eficiencia y de impacto de los programas gubernamentales en los problemas que trataban resolver. Los intentos de reformas, especialmente dirigidos a los procesos de planeación de los que la evaluación formó parte, comienzan con los trabajos realizados, desde 1965, por la Comisión de Administración Pública, a la que pertenecieron tanto el futuro Presidente José López Portillo (1976-1982) como varios colaborados suyos.

En resumen, puede decirse que, hasta el año 1999, las actividades de control y evaluación de la Administración Pública eran ejercidas por: la SHCP, la entonces Secretaría de la Contraloría y Desarrollo Administrativo (hoy Secretaría de la Función Pública, SFP) y la Contaduría Mayor de Hacienda de la Cámara de Diputados (hoy Auditoría Superior de la Federación, ASF). Se trataba fundamentalmente del control del gasto público, la legalidad de los procesos, el combate a la corrupción, entre otros., pero no se tiene conocimiento de que estas instancias evaluaran algún programa gubernamental, menos social, en términos de sus resultados o impactos.

En cuanto a la evaluación por un Poder Legislativo independiente, que tiene entre otras funciones la de servir de contrapeso al Poder Ejecutivo dentro de un marco democrático, cuestionando y generando información alternativa a la que el último aporta en forma unilateral, prácticamente no fue posible ejercerla durante el siglo pasado en México, debi- 
do a su composición de partido único, mismo al que también pertenecía el Presidente de la República, y a su consecuente dinámica de sumisión a la voluntad de este último. A partir de 1997, y más aún del 2000, esta situación comenzó a cambiar, al lograr cierto nivel de independencia de opinión y decisión, fruto de una composición más plural.

En este contexto, una de las tareas, constitucionalmente asignada y no desempeñada durante décadas, la constituye la evaluación del estado que guarda la Administración Pública Federal y los resultados alcanzados por las políticas públicas. Para cumplirla como actividad permanente de control de compromisos, resultados e impactos se requiere reactivar dos facultades básicas: los mecanismos formales de evaluación, control y fiscalización del ejercicio del presupuesto (revisión del gasto público, cumplimiento de metas anuales, entre otros.) y de la ejecución de planes, programas y proyectos (logro de objetivos de los programas, eficiencia en el uso de los recursos, entre otros) por parte de la Cámara de Diputados.

Adicionalmente, es necesario innovar en los mecanismos de rendición de cuentas a la ciudadanía, ya que los informes de gobierno ofrecen una imagen positiva de los avances realizados, al resaltar cuantitativamente las acciones implementadas en el marco de la planeación, sin referir su impacto real en la solu- ción de los problemas. Se trata de información parcial, por lo que no permiten una plena evaluación de sus planes y programas; mientras la glosa, uno de los instrumentos que utilizan las comisiones ordinarias de la Cámara en la evaluación del Plan Nacional de Desarrollo (PND) y los programas gubernamentales, ha sido más un elemento de refutación partidista que de evaluación real de resultados, y tiene un impacto muy bajo en la opinión pública.

Frente a situaciones como ésta, las democracias modernas han buscado mecanismos alternativos de participación ciudadana directa, como las incluidas en el actual PND mexicano o en los discursos de los organismos multilaterales, que requieren tener acceso a la información gubernamental.

En cuanto a la participación de las organizaciones de la sociedad civil, ninguno de los modelos tradicionales, burocrático o de mercado, han supuesto que los ciudadanos tengan un papel a jugar en la evaluación de las acciones del gobierno. También ellas descuidaron por un tiempo este aspecto, ya que "sufrían" la evaluación como parte de un proceso de control de los organismos que financian sus actividades, cuyos intereses y los de las elites nacionales se imponían contra toda evidencia, y la percibían con un alto costo de oportunidad para la realización de sus programas sustantivos ${ }^{2}$. Pero, vas y de investigación por el tema. Si bien fueron éstas las que iniciaron la realización de evaluaciones independientes, a la fecha no existen programas especialmente enfocados al desarrollo de esta incipiente profesión y puede constatarse el reducido número de tesis (16) vinculadas al tema que se ha podido localizar. 
mientras el nuevo sistema de rendición de cuentas cumple su objetivo, las organizaciones sociales y civiles, han avanzado en su conocimiento y práctica en procesos de evaluación.

El actual gobierno del Lic. Vicente Fox incluyó entre sus objetivos: "Establecer procesos de evaluación de las acciones de gobierno que ajusten su labor a las necesidades de la sociedad" (PND, 2001: 66). Ante todo, las políticas y los programas sociales se preveía que serían evaluados por la propia administración pública federal, quien integraría sus avances, logros y retrasos en ese mismo informe de evaluación del PND, que se prepara anualmente, de igual forma en que habría participado en el proceso de planeación (PND, 2001).

Las acciones en materia de política social se relacionan con la participación en los siguientes objetivos planteados en el plan nacional de desarrollo, que a continuación se sustentan :

- Evitar la existencia de grupos de población "...cuyas condiciones de vida, oportunidades de superación personal y de participación social, se encuentren por debajo de ciertos umbrales" (PND, 2001:50).

- Fomentar la actitud independiente y emprendedora de los ciudadanos, su capacidad e iniciativa, promoviendo proyectos que mejoren la preparación, la escolaridad y los conocimientos de la población, conduzcan al desarrollo de sus habilidades y destrezas, entre otros.

- Propiciar, conservar y alentar la cohesión social, aumentar la solidaridad de los mexicanos entre sí y con el bien común, entre otros, mediante políticas y proyectos incluyentes. En particular, fomentar la participación de las organizaciones sociales y civiles en el diseño, instrumentación y evaluación de las políticas y programas públicos.

- Recuperar la confianza en la capacidad del gobierno y las instituciones, mediante, entre otras acciones, el diseño de respuestas eficaces a situaciones no previstas de orden natural y social. Para su evaluación se prevé la utilización de indicadores como la rendición de cuentas, la calidad del trato a usuarios de los servicios públicos y el alcance de la protección financiera frente a eventos catastróficos.

El gobierno declaró su vocación de dialogar en materia de trabajo, desarrollo social, grupos vulnerables y competitividad, tanto con el Congreso de la Unión y el Poder Judicial, como con las autoridades de otros ámbitos de gobierno. Se esperaba que sus evaluaciones servirían como contrapeso del Poder Ejecutivo y se complementarían con la participación de la ciudadanía en estos procesos.

EI PND identifica importantes cambios en la participación social: mientras hasta la finalización de la década de los sesenta, las organizaciones de la sociedad civil eran sólo de carácter sindical o sectorial, a partir de esa fecha se ha multiplicado el número de organizaciones de la sociedad civil interesadas en los asuntos públicos (PND, 2001).

Como puede verse, la descripción que realiza el PND respecto de la planeación del gobierno foxista, da una imagen de alta participación ciudadana, fuertemente discutible. El mismo documento se compromete a pasar de la "amplia partici- 
pación ciudadana en la elaboración del PND a una amplia participación en los procesos de evaluación de resultados..." (PND, 2001: 45).

En relación con la rendición de cuentas, como ya se mencionó, la participación ciudadana requiere información, por lo que, un nivel mínimo de participación implica que los resultados de las evaluaciones se publiquen para que los interesados las conozcan, analicen, critiquen, discutan y propongan.

Las modificaciones aprobadas en 2001 permiten a la Cámara de Diputados analizar los resultados de los programas del año fiscal anterior antes de realizar las asignaciones de presupuesto para el siguiente. Dicha Cámara cuenta con diversas comisiones vinculadas al tema: Comisión de Desarrollo Social, Subcomisión de Evaluación, Comisión de Participación Ciudadana, Comisión de Presupuesto y Cuenta Pública, de Atención a Grupos Vulnerables, ASF, entre otros. También el Senado tiene una Comisión de Desarrollo Social y una de Atención a Grupos Vulnerables. Sus esfuerzos constituyen formas loables de empezar a introducir el tema pero, no aparece ningún mecanismo que informe a la población de las evaluaciones realizadas ni mucho menos que organice su participación en la realización de evaluaciones de los programas gubernamentales.

A partir del ejercicio fiscal 2001, la Cámara de Diputados comenzó a exigir la realización de evaluaciones externas a los programas que reciben recursos fiscales para controlar la obligación del gobierno federal de administrar con eficiencia, eficacia y honradez los recursos económicos que dispone para el cumplimien- to de objetivos. Para lograrlo exige la realización de licitaciones públicas (salvo excepciones), mediante convocatoria también pública a instituciones académicas y de investigación de reconocido prestigio, de manera de asegurar las mejores condiciones de precio, calidad, financiamiento, oportunidad y demás aspectos pertinentes.

En Junio de 2002 fue aprobada la Ley Federal de Transparencia y Acceso a la Información y se procedió a la creación del Instituto Federal de Acceso a la Información Pública (IFAI). Esta Ley preveía un plazo de un año para que todas las entidades obligadas pusieran su información al alcance del público; sin embargo, a la fecha, sólo se perciben avances parciales. Sin embargo, entre las obligaciones señaladas por la Ley de Transparencia no se incluye información sobre los resultados de evaluaciones realizadas. Esto a pesar de que la ASF resalta la importancia de la evaluación del desempeño en la rendición de cuentas, como forma de identificar desviaciones de los propósitos o deficiencias en su planteamiento $y$ funcionamiento.

A finales de 2003, se aprobó también la Ley General de Desarrollo Social, que promueve la evaluación y participación social y ciudadana en los programas sociales a través de instrumentos como la Contraloría Social. Un mes más tarde, se hizo lo propio respecto de la Ley Federal de Fomento a las Actividades Realizadas por Organizaciones de la Sociedad Civil.

Pero, ¿qué está pasando con las evaluaciones externas realizadas a programas sociales a partir de año 2001? Antes de la existencia de la Ley Federal de Transparencia y de Acceso a la Infor- 
mación, no existía ningún mecanismo que permitiera conocer sus resultados. Desde la entrada en vigencia de esta ley, es posible solicitar la información desde la página web del IFAI, la que debería ser entregada en un plazo de cuatro semanas. En 2003 fue posible consultar por INTERNET, en las páginas de los programas o de las instancias que los operan, los resúmenes de los reportes finales de cuatro programas (Desarrollo Humano Oportunidades, Abasto Social de Leche, Tortilla y Abasto Rural). Hasta la fecha los informes finales de evaluación no se han publicado; sin embargo, en el futuro deberán difundirse en el Diario Oficial, en cumplimiento de lo estipulado por la Ley General de Desarrollo Social, lo cual no será fácil debido al volumen que implica (200 páginas por informe, en promedio) y el costo que eso supone.

\section{Análisis de la evaluación de programas sociales}

A continuación se procede al análisis del conjunto de evaluaciones de los 31 programas sociales que la Secretaría de Desarrollo Social (SEDESOL) tuvo a su cargo durante el año 2002.

\subsection{Organización del sistema de evaluación}

Cada uno de los programas cuenta con una oficina encargada de efectuar las licitaciones, contratos, control de la entrega de informes convenidos, relacionarse con las instituciones y los equipos de investigadores que realizan las evaluaciones, enviar los informes finales a la SEDESOL y autorizar el pago al evaluador.
La SEDESOL realiza comentarios a los evaluadores y entrega los informes a la Cámara de Diputados, SHCP y SFP.

Pero, más allá de la organizacional formal del sistema, interesa analizar las razones que llevaron a la decisión de la Cámara de Diputados de evaluar anualmente todos los programas, la manera en que se aplican efectivamente los procedimientos, $y$ los intereses que mueven a los actores participantes.

En relación al primer punto, la SEDESOL considera que se trató de una iniciativa de los operadores de los propios programas y de la SHCP, esta última sentía la necesidad de controlar a los distintos ámbitos de gobierno de diferentes adscripciones partidistas, misma que fue compartida y apoyada por la Cámara de Diputados. Sin embargo, no hay discusión alguna que dé sustento a las posiciones de los diputados, ya que los diarios de debate de la época no mencionan que el asunto haya causado polémica alguna.

Algunos académicos de relevancia consideran que es la crisis fiscal la que ha venido preocupando desde los ochentas a la SHCP y que, en su afán por controlar el gasto gubernamental, ha querido realizar evaluaciones que le permitan recortar el gasto social improductivo. Algo muy similar se ha sugerido en el contexto francés para justificar su presencia: romper las tendencias anteriores de crecimiento de las prestaciones de servicio público y lograr su aceptación por la sociedad (Duran y Monnier, 1992).

Los Términos de Referencia para la evaluación externa establecen las características del producto esperado, así como los requisitos para la participación de los equipos interesados en realizarla. 
Su redacción es casi idéntica en todos los casos, presentándose muy poca consideración de los aspectos específicos de cada programa, y dejando algunas ambigüedades o induciendo confusiones, al mismo tiempo que se muestra muy ambiciosa en casos que no lo justifican. La respuesta mayoritaria de los evaluadores en esta situación ha sido ofrecer lo que se pide, a pesar de que a menudo no podrán satisfacerlo, e intentar llenar el punto con un discurso bastante vacío de contenido. Es un juego en que se piden imposibles, se responde como si se hubieran cubierto y quienes hacen la revisión no exigen congruencia.

Los procedimientos de convocatoria y selección de evaluadores se han llevado a cabo con relativa normalidad. De acuerdo con la ley de Adquisiciones, Arrendamientos y Servicios del Sector Público, se debe realizar un concurso público para contratar a organizaciones privadas, salvo casos de excepción. En la práctica de la evaluación se ha venido aplicando el procedimiento de invitación "a cuando menos tres organizaciones", salvo que la evaluación sea efectuada por una organización del propio sector gubernamental y se considere que no existe otra con infraestructura, personal y capacidad suficiente para realizarla, en cuyo caso se procede a la adjudicación directa. Este último procedimiento se ha utilizado en el caso de la evaluación del programa Oportunidades y se habría intentado aplicarlo nuevamente para la adjudicación directa de las evaluaciones multianuales programadas, provocando un profundo descontento en evaluadores externos que desean participar en su realización.
En el caso del procedimiento por invitación, se forma una comisión integrada por miembros de SEDESOL y de la instancia responsable del programa, la cual analiza las propuestas recibidas que cubren todos los aspectos solicitados y elige la más económica, de acuerdo con el criterio especificado en la Ley de Adquisiciones, Arrendamientos y Servicios del Sector Público (2000). Esto provoca serios problemas ya que la comisión no puede tomar en cuenta criterios vinculados a la calidad estimada del trabajo, junto con los costos presupuestados.

Como el mercado de la evaluación está aún en proceso de conformarse, sucede en algunos casos, que no hay instituciones interesadas en realizar la evaluación. En estas situaciones, después de dos convocatorias públicas o por invitación, también se puede efectuar una adjudicación directa de la evaluación a alguna organización que se considere podrá cumplir las expectativas.

Muchas instituciones e investigadores de reconocido prestigio se niegan a participar por considerar que los recursos adjudicados son muy limitados y que se requiere realizar un excesivo papeleo burocrático. Por otro lado, existen muchos pequeños centros en crecimiento, interesados en obtener proyectos que les permitan continuar en el mercado y dispuestos a sacrificar parte de sus posibles ganancias con tal de obtener el contrato. Esto ha provocado que, en 2002 y 2003 , los recursos disponibles para remunerar las evaluaciones realizadas sean bastante superiores (hasta $40 \%$ ) a los efectivamente asignados.

Como resultado de los procesos revisados, es posible afirmar que las asig- 
naciones realizadas cubren, en términos generales, con los requerimientos establecidos en los Términos de Referencia. Sin embargo, preocupa que las excepciones a esta regla se produzcan en casos en que el presupuesto destinado a la evaluación resulta de los más cuantiosos. Lo anterior, unido a que el pago de la evaluación es realizado por la misma instancia que opera el programa, pone en riesgo la independencia de criterio del evaluador.

El móvil que induce a la participación institucional revisada es fundamentalmente la obtención de experiencia y reconocimiento a su trabajo $\mathrm{y}$, en mucho menor grado y sólo en algunos casos, los ingresos recibidos ${ }^{3}$.

Algo similar sucede con los coordinadores del equipo de evaluadores, que si bien reciben ingresos adicionales, a veces sustantivos, realizan el trabajo en búsqueda, principalmente, de experiencia y reconocimiento. Sin embargo, hay que destacar la existencia de un grupo de asistentes de investigación y encuestadores para los cuales la evaluación llega a constituir una oportunidad única de lograr ingresos, en el contexto de insuficiencia y precarización creciente del empleo en el país. Se constató en varios casos que los últimos pueden llegar a impulsar a los coordinadores a participar.

\subsection{Recursos para la realización de evaluaciones}

El caso del programa de Desarrollo Humano Oportunidades (en adelante
Oportunidades) resulta absolutamente extraordinario por sus altos montos, tanto de presupuesto ejercido, como de costo de sus evaluaciones (aprox. 540 y $2.6 \mathrm{mi}$ llones de dólares, respectivamente). Los siguientes presupuestos más elevados corresponden a cuatro programas con un promedio de 94 millones de dólares y los siguientes costos de evaluación a cinco programas con un promedio de $0.17 \mathrm{mi}$ llones de dólares. No se perciben correlaciones fuertes entre los presupuestos ejercidos por los programas y los costos de sus evaluaciones.

En cuanto a la calificación y experiencia de los recursos humanos del sistema de evaluación, comenzaremos por quienes forman parte de la Dirección General de Evaluación en SEDESOL y los funcionarios de las instancias operadoras, encargados de la contratación de evaluadores.

En relación a los primeros se nos manifestó en entrevista que se está privilegiando la evaluación de impacto económico, por lo que casi todos cuentan con formación en esa disciplina, graduados en su mayoría en una institución educativa privada ${ }^{4}$, con posgrados en instituciones extranjeras de excelencia, en áreas de Econometría, Evaluación de Programas Sociales, Políticas Públicas, entre otros. Su formación explica el énfasis econométrico que parece imponerse como criterio de calidad. La mayoría de esos especialistas cuenta con experiencias relevantes de trabajo previo, aunque no necesariamente en evaluación.

3 Estos varían entre el 10 y el 30\% del monto contratado.

4 Instituto Tecnológico Autónomo de México (ITAM) 
En opinión de varios evaluadores externos, la mayoría de quienes se responsabilizan de la contratación de evaluadores para cada programa no están suficientemente capacitados para calificar las propuestas técnicas y asignar los contratos.

La composición de los equipos evaluadores en 2002, con base en la información que ofrecen los informes y la recogida mediante entrevistas, resulta muy variada. La información disponible es insuficiente para realizar juicios categóricos; sin embargo, de los 17 programas que presentan alguna información encontramos que sólo siete cuentan con equipos sólidos en términos de experiencia y formación multidisciplinaria pertinente en el caso.

Adicionalmente, los recursos humanos destinados a la revisión de los informes en la SHCP y la SFP son poco numerosos (no más de 6 personas) y sólo dedican parte de su tiempo a esta actividad.

\subsection{Fuentes de información y tratamiento de los datos}

Los Términos de Referencia señalan, en forma general, los métodos de recolección y tratamiento de datos, que en todos los casos revisados incluyen trabajo de gabinete, con base en la información secundaria disponible, y trabajo empírico. Además se deberían emplear métodos de análisis costo-beneficio, costoeficacia, entre otros., lo que casi no se intenta en la práctica.

Casi la totalidad de las 31 evaluaciones revisadas incluyeron, entre sus fuentes de información, documentos y bases de datos estadísticos, y aplicaron encuestas y entrevistas, con pleno cumplimiento de los lineamientos normativos revisados.

Respecto de la información secundaria entregada por la autoridades de los programas, casi la mitad de los evaluadores manifiestan diversos problemas: insuficiencia, poca confiabilidad, mala calidad y falta de oportunidad.

En el caso de las entrevistas, éstas se dirigen a informantes-clave (funcionarios $y$, en menor medida, miembros de las comunidades de beneficiarios), mediante una selección especialmente dirigida a quienes disponen de información privilegiada.

Por el contrario, en el caso de las encuestas con pretensiones de representatividad (como sucede en la mayoría de la evaluaciones), éstas requieren de la correcta determinación del tamaño muestral y la composición de la misma, de manera que todos los individuos del universo tengan igual probabilidad de ser seleccionados mediante la aplicación sistemática de algún método de muestreo (aleatorio simple, aleatorio estratificado, por conglomerados, entre otros).

Cabe señalar que, si bien en determinadas condiciones no se toma en cuenta el tamaño del universo para la determinación del correspondiente a la muestra, el listado del mismo facilita la composición aleatoria de ésta. Sin embargo, dicha información no se encuentra disponible en la mayoría de los casos. Esta situación se agrava debido a la ausencia casi total de exposición del método empleado en su determinación, con excepción de algunos informes.

Sólo siete de los programas analizados disponen de la cuantificación de su 
población objetivo, misma que, en varios casos, coincide aproximadamente con la estimación realizada por SEDESOL de 19 '225,833 habitantes en las 263 regiones prioritarias definidas, lo que muestra que no ha sido calculada específicamente para el programa en cuestión. En la tercera parte de las evaluaciones no se presenta información completa sobre el tamaño muestral y sólo en dos es posible conocer el porcentaje de beneficiarios que fue encuestado y así contar con una idea aproximada de su nivel de representatividad. Éste resulta sumamente bajo, variando entre dos y seis encuestados por cada 100,000 personas.

Concluyendo sobre este aspecto, puede afirmarse que el fundamento metodológico de lo que se reporta en los informes, sobre la base de la aplicación de encuestas, resulta de una debilidad muy grande.

En cuanto al tratamiento de la información, prácticamente todos los programas combinan el análisis cuantitativo y el cualitativo, por lo que sólo pueden plantearse diferencia de énfasis. Sólo se presentan tres informes en que el trabajo es fuertemente descriptivo, limitándose a presentar cuadros y gráficas que ordenan los datos, en lugar de ser más analítico. Son también muy pocos los que intentan aplicar algunos métodos específicos, los que en varios casos no resultan claros o definitivamente presentan problemas en la selección de la información utilizada en el cálculo.

En resumen, se evidencia un énfasis en la aplicación de entrevistas y encuestas de representatividad pocas veces fundamentada, que se complementa con un tratamiento medianamente aceptable de los datos recogidos.

\subsection{Contenido de la evaluación}

Los Términos de referencia solicitan: 1) análisis de los costos y gastos de operación y el cumplimiento de metas financieras, 2) apego a la normatividad del programa, 3) análisis desglosado de la cobertura y su variación respecto del año anterior, 4) cumplimiento de las metas físicas, 5) determinación de beneficios económicos y sociales provocados por el programa, 6) satisfacción de los beneficiarios, 7) opinión sobre la permanencia o conclusión del programa, conclusiones y recomendaciones, y, 8) escenarios prospectivos.

En cambio no solicitaban en 2002: 1) estudio de la pertinencia y diseño del programa, 2) disposición de recursos no financieros, 3) evaluación de los procesos de gestión operativa del programa, 4) impacto logrado por el programa en la solución del problema que le dio origen, 5) participación de los beneficiarios y otros grupos sociales en la gestión y evaluación del programa, 6) utilización de resultados de evaluaciones previas, y, 7) emisión de un juicio valorativo sistemáticamente fundamentado sobre la gestión del programa y sus logros.

Los ocho aspectos solicitados son cubiertos en distintos grados por los 31 informes de evaluación, sugiriendo los siguientes comentarios:

- Un análisis completo de los costos y gastos de operación y el cumplimiento de metas financieras debería hacer alusión al gasto total programado, y al ejercido, su evolución en los últimos años y su distribución por tipo de apoyo, estados, entre otros., así como su monto promedio por beneficiario y población objetivo. Sin embargo, no to- 
dos estos elementos se solicitan, por lo que muchos informes incluyen información parcial al respecto.

- En cuanto al apego de los programas a su normatividad y la transparencia de su operación, existe mucha dificultad para su verificación por el evaluador, lo que sólo puede hacerse en función de las percepciones de la muestra seleccionada o mediante el estudio de expedientes. Con base en los últimos, es posible revisar aleatoriamente los resultados de dichos procesos, como por ejemplo la verificación de los requisitos de elegibilidad exigidos entre los beneficiarios encuestados, pero no los procesos mismos en que se tomaron las decisiones.

- La expectativa respecto de la medición de cobertura resulta bastante ambiciosa, requiere disponer de buenas fuentes de información institucional, ya que el evaluador sólo puede verificar la cobertura en los lugares seleccionados para la realización de trabajo de campo. Si el dato no existe, el evaluador no puede obtenerlo porque éste requiere de un trabajo censal. En casi todas las evaluaciones, se incluye la cantidad de población atendida como cálculo de la cobertura, aún cuando lo más importante es su porcentaje, que relaciona la población anterior con la población objetivo total, y permite valorar el esfuerzo que aún resta por hacer.

- En general, hay un tratamiento suficiente de los servicios brindados y el cumplimiento de metas físicas, con pocas excepciones. Es muy restringido el número de informes que profun- dizan en el análisis de su evolución y distribución. Algunos analizan críticamente tanto la información que se solicita como los indicadores propuestos para su sistematización.

- En cuanto a la satisfacción de los beneficiarios, ésta es recogida en la mayoría de las evaluaciones mediante la aplicación de encuestas; sin embargo una tercera parte de las mismas no la revisan. Lo anterior muestra poco apego de los evaluadores a un aspecto solicitado, importante y fácil de cubrir.

- Son muy pocas las evaluaciones que incluyen escenarios prospectivos, por lo que constituye el aspecto menos cubierto de todos los solicitados y hace suponer que, o bien los evaluadores no tuvieron tiempo suficiente para realizarlo, ya que es el último paso del proceso solicitado; o bien hay muy poca expertise desarrollada en el tema.

En cuanto a los siete aspectos no solicitados en la evaluación corresponde resaltar de todas formas algún tratamiento del tema por parte de los evaluadores:

- En 2002, los Términos de Referencia suponían implícitamente que los programas están bien diseñados, por lo que sólo valía la pena evaluar sus resultados e impactos. En consecuencia pocos informes evaluaron la magnitud de las necesidades sociales que justifican el programa, su consistencia con el PND y el Programa Nacional de Desarrollo Social, el diseño de sus objetivos o la congruencia entre estos y las estrategias del programa. El aspecto más desarrollado fue el de su focalización, que constituye la 
principal estrategia aplicada a los programas de superación de la pobreza.

- De importancia relevante para el funcionamiento del programa son también los recursos no financieros, tales como los tradicionales recursos humanos y materiales (instalaciones, muebles, equipos, entre otros.), así como los menos tradicionales, vinculados a la información y la capacidad gerencial, que se incluyeron en pocos casos.

- Los procesos de gestión desarrollados quedan ocultos en una "caja negra" cuya revisión no parece interesar a los solicitantes en aspectos como la productividad, calidad y eficiencia con que los insumos son transformados en productos o servicios. A pesar de ello, todos los evaluadores incluyen referencias a los mismos y a los principales problemas identificados. Este análisis podría homogeneizarse, para facilitar comparaciones, mediante un guión de aspectos a considerar, sin perder de vista las particularidades de cada programa evaluado.

- Provoca dificultades, tanto por falta de información como por la dificultad de su tratamiento, el intento por identificar y medir los impactos, más allá de los beneficios económicos y sociales solicitados. Oportunidades es el único programa que se arriesga a dar una cifra de población que superó la pobreza externa $(5 \%)$, misma que ha sido muy discutida en el medio académico y periodístico. En este trabajo no se considera realista la medición anual de impacto que se pretende realizar.
- El seguimiento de las recomendaciones realizadas en evaluaciones previas permitiría dar continuidad a los trabajos realizados y detectar su utilidad práctica. Sin embargo, la tercera parte de los programas evaluados no lo refieren, presentándose como un esfuerzo aislado. En las entrevistas complementarias realizadas destaca la nula utilización de resultados para la determinación del presupuesto por la Cámara de Diputados, su empleo limitado para el ajuste de los programas por las entidades responsables y para el mejoramiento de la normatividad y términos de referencia en el caso de SEDESOL. Lo anterior muestra una tendencia al mero cumplimiento formal en la realización de las evaluaciones externas.

- Toda evaluación supone, para ser considera como tal, la elaboración final de un juicio valorativo, sistemáticamente fundamentado, sobre la gestión del programa y sus logros. Siendo rigurosos sólo la tercera parte de los trabajos cumplen con este requisito para ser consideradas verdaderas evaluaciones y existen tres casos absolutamente insuficientes en materia de conclusiones.

\subsection{Participación de los beneficiarios y organizaciones sociales}

La participación social y ciudadana es una de las estrategias privilegiadas por el PND; sin embargo, los Términos de Referencia sólo la solicitan expresamente en dos de los programas evaluados. A pesar de ello, casi la mitad de los informes mencionan la ausencia de participa- 
ción o a los bajos niveles alcanzados por la misma:

- En la gestión de los programas, hay referencias a aportaciones de dinero o trabajo, pero éstas son mínimas en lo que atañe a la participación en la toma de decisiones.

- En casi todos los programas hay una participación mínima de los beneficiarios, y en algunos casos de otros actores sociales, pero sólo en calidad de informantes, mediante la respuesta a encuestas y entrevistas.

- No hay ninguna referencia a la participación social y ciudadana en el análisis y valoración de los procesos, resultados e impactos de los programas.

La situación anterior fue confirmada por los diversos actores entrevistados: Director de Metodología de Evaluación de SEDESOL, evaluadores, miembros de SHCP y SFP y del Poder Legislativo.

Ante esta falta de participación de los beneficiarios en la toma de decisiones y los procedimientos de evaluación, surgió la inquietud de investigar si alguna organización de la sociedad civil participaba en los procesos de evaluación gubernamental. Ninguna de las quince organizaciones y consultoras especializadas en ellas que fueron entrevistadas había tenido relación alguna con los procesos de evaluación anual externa de programas gubernamentales en estudio. Sólo se localizó una que había sido contratada para realizar otro tipo de evaluación a un programa gubernamental y otra que realizó una evaluación independiente al programa antecesor de Oportunidades (PROGRESA), dentro de la campaña "El Banco Mundial en la Mira de las Mujeres".
En conclusión, puede afirmarse que la participación de los beneficiarios y las organizaciones de la sociedad civil es mínima en el contexto de la gestión y evaluación de programas sociales.

\section{Juicio valorativo del análisis y propuesta de clasificación de evaluaciones}

Como resultado de la metaevaluación realizada, en términos generales, encontramos un panorama en que los recursos institucionales (presupuestales y de información) presentan limitaciones importantes para el desarrollo de trabajos de calidad y un mercado de evaluaciones aún en formación, contando con una oferta, también restringida, de capacidades especializadas en el tema. A esto se agregan imprecisiones y ausencias por parte de la demanda (Términos de Referencia). En este contexto, y salvo pocas excepciones, se desarrollan evaluaciones con base en la aplicación de instrumentos a muestras de representatividad desconocida o dudosa, se cubren parcialmente los aspectos solicitados, y otros de reconocida relevancia para arribar a conclusiones bien fundamentadas (aunque no hayan sido solicitados); y se efectúan pocas valoraciones explícitas del programa analizado.

Se insiste en que el juicio anterior no se dirige exclusivamente a la calidad del trabajo de los evaluadores, sino a la alcanzada por el informe final presentado, misma que depende de diversos factores, algunos de los cuales son de responsabilidad gubernamental.

A pesar de la generalidad del juicio pronunciado, hay que reconocer diferen- 
cias entre el conjunto de evaluaciones, lo que nos lleva a proponer una clasificación del mismo, con base en el presupuesto destinado al trabajo y la calidad de su producto. Como indicador de la última, y con todas las limitaciones que implica utilizar sólo información seleccionada, se realizó una combinación de cuatro de los elementos revisados en esta metaevaluación: 1) haber sido realizada por un equipo interdisciplinario, pertinente al tema del programa evaluado, 2) contar con un diseño, recolección y tratamiento de datos riguroso, 3) cumplir con los requerimiento especificados en los Términos de Referencia, y 4) concluir con la explicitación de un juicio valorativo sobre el programa.

Como resultado de lo anterior se obtienen tres categorías que permiten identificar seis evaluaciones de alta calidad, seguidas de otras once de calidad aceptable, y las trece restantes con baja calidad (una no se calificó por tratarse de un programa casi sin actividad cuya evaluación es muy acotada). Se aclara que ninguna de las evaluaciones consideradas cumplió plenamente con todos los parámetros considerados, razón por la que no se alcanzó un nivel que pudiera calificarse como óptimo.
Puesto que no sería justo medir con la misma vara la calidad de evaluaciones que contaron con montos de recursos muy diferentes para su realización, el estudio combinado de ambos aspectos se presenta en la siguiente Tabla 1.

De la Tabla 1 se desprenden los siguientes comentarios:

- Quince casos muestran congruencia entre los recursos aplicados y la calidad lograda.

- El nivel más alto de presupuesto corresponde ampliamente al programa Oportunidades; sin embargo, a pesar de su alta calidad, no es posible juzgarla como óptima, debido a que no cumple con algunos de sus requisitos establecidos. Por otra parte, se considera que el diseño de grupos de tratamiento y control a nivel de escuelas y centros de salud no resulta suficiente para medir impactos, en que se debería trabajar a nivel de los beneficiarios. Tampoco se integran los diversos trabajos realizados en una evaluación única, ni se emite un juicio valorativo de conjunto.

- Resulta preocupante que tres evaluaciones de alto costo logren sólo un nivel bajo de calidad.

\section{Tabla 1. Relación entre recursos y calidad de las evaluaciones de Programas Sociales en México (número de evaluaciones)}

\begin{tabular}{lccccc}
\hline & $\begin{array}{c}\text { Recursos } \\
\text { bajos }\end{array}$ & $\begin{array}{c}\text { Recursos } \\
\text { medios }\end{array}$ & $\begin{array}{c}\text { Recursos } \\
\text { altos }\end{array}$ & $\begin{array}{c}\text { Recursos } \\
\text { muy altos }\end{array}$ & Total \\
\hline Calidad baja & 8 & 2 & 3 & 0 & 13 \\
Calidad media & 4 & 6 & 1 & 0 & 11 \\
Calidad alta & 0 & 4 & 1 & 1 & 6 \\
Total & 12 & 12 & 5 & 1 & 30 \\
\hline
\end{tabular}

Fuente: elaboración propia a partir del análisis de los informes de evaluación de cada programa. 
- Existe un grupo de cuatro programas que recibió niveles medios de recursos y logró resultados cuya calidad sobresale en ese conjunto.

- Finalmente, tenemos que resaltar otro pequeño grupo que, con bajos recursos, logra una calidad aceptable en su trabajo.

\section{Conclusiones}

Tanto la teoría de la gerencia pública (especialmente el NPM) como el discurso político insisten en la conveniencia de la evaluación y participación social y ciudadana. Sin embargo, AL cuenta con avances muy restringidos en materia de evaluación de la acción pública y participación social, que contrastan con el discurso reinante. En el caso de México esto resulta altamente incongruente con la participación efectiva que describimos para la gestión de los programas, donde se enfatiza la aportación de dinero y trabajo, por sobre la integración a la toma de decisiones. En materia de rendición de cuentas sobre los resultados e impactos logrados por los programas sociales resta mucho terreno por andar; sin embargo, la evolución revisada en México muestra avances en los últimos años. En relación con la evaluación de programas sociales, México ha realizado progresos importantes a partir de 2001, pero la calidad alcanzada aún enfrenta problemas diversos y no se correlaciona con el presupuesto invertido en su realización. No existe ningún programa mexicano que prevea la participación social y ciudadana en los procesos de análisis y valoración del mismo, limitándose a considerar a la ciuda- danía como mero objeto de encuestas y entrevistas, en calidad de informante. Es urgente que las universidades de AL formen evaluadores con conocimiento del enfoque participativo, especialmente en el caso de programas que apoyen el desarrollo económico-social, con carácter sustentable.

\section{Referencias Bibliográficas}

Bañón i Martínez, Rafael, Comp. (2003), La evaluación de la acción y de las políticas públicas, Madrid, Díaz de Santos.

Brown, Deryck (Enero de 2001), "Evaluación de políticas y programas de la región del Caribe de habla inglesa: problemas conceptuales y prácticos", Serie Gestión Pública, No.9, Santiago de Chile, CEPAL - ILPES.

Congreso de la Unión (4 de Enero de 2000), Ley de Adquisiciones, Arrendamientos y Servicios del Sector Público, Diario Oficial de la Federación.

Congreso de la Unión (2002), Ley Federal de Acceso a la Información Pública Gubernamental, México: www.secodam.gob.mx, consulta efectuada el 2 de Mayo de 2003.

Congreso de la Unión (2003), Ley General de Desarrollo Social: www.sedesol.gob.mx, consulta efectuada el 31 de Marzo de 2004.

Congreso de la Unión (2004), Ley Federal de Fomento a las Actividades Realizadas por las Organizaciones de la Sociedad Civil: www.cddhcu.gob.mx, consulta efectuada el 22 de Julio de 2004.

Cunill, Nuria y Sonia Ospina (2003), Evaluación de resultados para la gestión pública moderna y democrática: experiencias latinoamericanas, $V e$ nezuela, CLAD-AECI-MAP-FIIAPP. 
Duran, Patrice y Monnier E. (Abril 1992), "Le développment de l'évaluation en France. Nécessités techniques et exigences politiques", en Revue Française de Science Politique, Vol. 40, No. 2, Francia, pp.235-262.

Kliksberg, Bernardo (1989), “¿Cómo Formar Gerentes Sociales? Elementos para el Diseño de Estrategias", en B. Kliksberg (comp.) ¿Cómo enfrentar la pobreza?, Argentina, Grupo Editor Latinoamericano.

Kliksberg, Bernardo (1994), Pobreza. El drama cotidiano. Clave para una nueva gerencia social eficiente, Venezuela, CLAD, PNUD, Edit. Tesis-Norma.

Kliksberg, Bernardo (2000), "Seis tesis no convencionales sobre participación", en Acta Sociológica, Nos. 28-29, México, UNAM, pp. 87-124.

Licha, Isabel (1998), Participación comunitaria, mimeo, EUA, INDES-BID.

Monnier, Eric (1991), "Objectifs et destinataires des évaluations", en Documentación Administrativa, №224-225, España, pp.131-157.
Monnier, Eric (1995), Evaluación de la acción de los poderes públicos, España, Instituto de Estudios Fiscales.

Ospina, Sonia (2001), "Evaluación de la gestión pública: conceptos y aplicaciones en el caso latinoamericano, en Revista Reforma y Democracia, No. 19, Venezuela, CLAD, pp. 91-122.

Plan Nacional de Desarrollo 2001-2006, en www.sedesol.gob.mx, consulta efectuada el $1^{\circ}$ de mayo de 2003.

SEDESOL (2003), 31 informes de evaluación de programas sociales, CD entregado por el IFAI.

Sulbrandt, José (1993), "La evaluación de los programas sociales: una perspectiva crítica de los modelos actuales", en Kliksberg, Bernardo (comp...), Pobreza. Un tema impostergable. Nuevas propuestas a nivel mundial, México, CLAD- FCE- Programa de Naciones Unidas. 